\title{
Liquid nitrogen cryotherapy vs. Betamethasone lotion in the management of Alopecia areata
}

\author{
Faghihi Gita $^{1 \star}$ and Radan Mohammadreza ${ }^{2}$ \\ ${ }^{1}$ Department of Dermatology, Skin and Leishmaniasis Research Center, Isfahan University School of Medicine, Isfahan \\ University of Medical Sciences, Iran. \\ ${ }^{2}$ Supervision Council in Clinical Practice, Isfahan University of Medical Sciences, Iran.
}

Accepted 25 October, 2011

\begin{abstract}
Alopecia areata (AA) is a common disease of hair follicles. Cryotherapy has been employed to stimulate hair regrowth. We decided to evaluate the efficacy of this method in patchy AA. Forty patients of age $>5$ years and with 120 recalcitrant patches were enrolled in this study. AA had covered less than $25 \%$ of patients' scalps. In each individual, one lesion was treated with nitrogen cryotherapy once weekly for a period of 6 weeks; meanwhile another lesion was treated with topical $0.1 \%$ betamethasone lotion. All patients were followed up from weeks 2 to 14. Thirty-nine out of forty patients completed therapy. The overall response rate was $\mathbf{8 8 \%}$ for patches treated with cryotherapy and $90 \%$ for patches treated with betamethasone lotion. Complete recovery ( $>90 \%$ terminal hair growth) was not obtained in any patient. Chi-square test showed no significant difference between improvements of alopecia in the two groups (reliability 95\%). Pearson measurement showed a significant reverse relationship between diameter and duration of patches and the improvement rate in both groups. Liquid nitrogen cryotherapy can be a helpful modality in the treatment of AA.
\end{abstract}

Key words: Alopecia areata, treatment, liquid nitrogen cryotherapy, betamethasone lotion.

\section{INTRODUCTION}

Alopecia areata $(\mathrm{AA})$ is a chronic inflammatory disease of the hair follicle usually manifesting as round or ovoid patchy areas that show hair loss with discrete borders. AA is common but unfortunately has no uniformly successful form of therapy (Schwartz and Janniger, 1997; McElwee, 1999). It is estimated to affect almost $2 \%$ of the U.S. population (Safavi et al., 1989). Mild limited involvement of the scalp is the most common presentation; multiple patches may become confluent over time. Regression may occur, with new hair growth taking place; recurrences in different locations occur. More severe forms of the disorder, involving the entire scalp, eyebrows, eyelashes, axillary, pubic areas or the entire body also exist (Madani and Shapiro, 2000; Sharma et al., 1996; Shapiro and Madani, 1999).

\footnotetext{
*Corresponding author. E-mail: g_faghihi@med.mui.ac.ir.
}

Alopecia areata (AA), especially when severe, often profoundly affects the lives of those afflicted. Patients with AA that have a history of atopy may have a less favorable prognosis (Tosti et al., 1994).

Current investigative efforts strongly implicate CD4 and CD8 T-cell lymphocytes in the etiology of this disorder. The $\mathrm{CD}^{+}$killer T cell is an effector cell which causes hair bulb injury, triggering the AA (Todes-Taylor et al., 1984; McElwee, 1999).

Though autoantibodies are postulated to play an integral role in the disease process, current research implicates a cell-mediated autoimmune mechanism as the underlying pathogenic etiology. CD44v10 is believed to be involved in the activation mechanism of CD4 and CD8 lymphocyte migration into tissue and the initiation of the subsequent defense response against antigenic stimuli (Gilhar et al., 1998). Supporting this theory is the fact that activated CD4 and CD8 T lymphocytes have been found in a characteristic peri- and intra-follicular 
Table 1. The improvement rate of lesions in the cryotherapy group.

\begin{tabular}{cc}
\hline Number of lesions (\%) & Terminal hair growth (\%) \\
\hline 0 & $<90$ \\
23 & $75-90$ \\
33.5 & $50-75$ \\
31.5 & $25-50$ \\
12 & $>25$ \\
\hline
\end{tabular}

Table 2. The improvement rate of lesions in the Betamethasone group.

\begin{tabular}{cc}
\hline Number of lesions (\%) & Terminal hair growth (\%) \\
\hline 0 & $<90$ \\
28 & $75-90$ \\
34.5 & $50-75$ \\
27.5 & $25-50$ \\
10 & $>25$ \\
\hline
\end{tabular}

inflammatory infiltrate of anagen hair follicles.

\section{MATERIALS AND METHODS}

\section{Inclusion criteria}

1. Age $>5$ years.

2. AA coverage less than $25 \%$ of scalp area.

3. Presence of at least two symmetrical patches over the scalp.

4. Maximum diameter of each lesion less than $7 \mathrm{~cm}$.

5. Absence of any other severe medical illness.

6. No simultaneous immune-suppressive use and discontinue of any other treatment at least 4 weeks earlier.

\section{Exclusion criteria}

1. Pregnancy.

2. Lactation.

3. Any newly onset medical systemic illness.

4. Progression of AA into more than $25 \%$ of scalp area.

5. Severe hemorrhagic bulla or any documented hypersensitivity to each of the procedures.

In each individual, one lesion was treated with nitrogen cryotherapy once weekly for a period of 6 weeks. The patients were matched by their ages and sexes. A cryogun was used to spray the liquid nitrogen to the area for 2 to $3 \mathrm{~s}$, until it became slightly frozen. After the frozen area thawed (about 3 to $5 \mathrm{~s}$ ), a second spray was done in the same manner. Meanwhile another lesion on the same patient was treated by topical $0.1 \%$ betamethasone lotion twice daily for 6 weeks. All patients signed the consent and were followed up from weeks 2 to 14 , fortnightly.

The side effects and amount of hair regrowth during the study were recorded. The evaluation was performed with clinical examination. According to the extent of terminal hair growth, the lesions were grouped into 4 categories:
1. Good response (regrowth of $>75 \%$ terminal hair).

2. Moderate response (regrowth of 50 to $75 \%$ terminal hair).

3. Poor response (regrowth of 25 to $50 \%$ terminal hair).

4. No response (regrowth of less than $25 \%$ terminal hair).

\section{RESULTS}

Thirty-nine out of forty patients (17 men, 22 women) completed the study. The patients' ages ranged from 9 to 58 years. The mean age was $22 \pm 2.5$ years. The duration of disease ranged from 3 months to 10 years. The average disease duration was 8 months. The two groups were not significantly different in their demographic characteristics, and in the sizes and patterns of patches at baseline (Tables 1 to 4 ).

The average diameter of lesions in cryo group was not statistically different from that of the other group, and was 3 to $3.5 \mathrm{~cm}$. The partial hair regrowth was seen in $88 \%$ of patches in the cryotherapy group and $90 \%$ of patches in the other group. No one attained full hair regrowth in each group.

One patient $(2.5 \%)$ discontinued the treatment by cryotherapy due to pain during cryo spraying and dissatisfaction.

The results in lesions treated with cryotherapy, were composed of:

1. Good response $23 \%$ (regrowth of $>75 \%$ terminal hairs).

2. Moderate response $33.5 \%$ (regrowth of 50 to $75 \%$ terminal hairs).

3. Poor response $31.5 \%$ (regrowth of 25 to $50 \%$ terminal hairs).

4. No response $12 \%$ (regrowth of less than $25 \%$ ).

And in the other group of lesions treated by topical betamethasone the results were as follows:

1. Good response $28 \%$ (regrowth of $>75 \%$ terminal hairs).

2. Moderate response $34.5 \%$ (regrowth of 50 to $75 \%$ terminal hairs).

3. Poor response $27.5 \%$ (regrowth of 25 to $50 \%$ terminal hairs).

4. No response $10 \%$ (regrowth of less than $25 \%$ ).

A comparison of improvement rate of the results of the two groups showed no significant difference $(p=0.75)$ with Chi-Square test (reliability co-efficiency $95 \%$ ).

The response of lesions according to the duration of disease was as follows:

\section{Cryotherapy group}

\section{Good response}

1. The lesions below 6 months: $46.5 \%$ 
Table 3. The relationship between lesion diameter and response rate in the cryotherapy group.

\begin{tabular}{|c|c|c|c|}
\hline \multirow{2}{*}{ Percentage of improvement } & \multicolumn{3}{|c|}{ Lesion diameter } \\
\hline & $<2.5 \mathrm{~cm}$ & $2.5-5 \mathrm{~cm}$ & $>5 \mathrm{~cm}$ \\
\hline Good response (Growth of more than $75 \%$ of terminal aesthetically acceptable hair) & $45 \%$ & $35.5 \%$ & $14 \%$ \\
\hline
\end{tabular}

Pearson measurement test analysis: $(P=0.03)$; $r=-0.25$.

Table 4. The relationship between lesion diameter and hair regrowth rate in the betamethasone group.

\begin{tabular}{|c|c|c|c|}
\hline \multirow{2}{*}{ Percentage of improvement } & \multicolumn{3}{|c|}{ Lesion diameter } \\
\hline & $<2.5 \mathrm{~cm}$ & $2.5-5 \mathrm{~cm}$ & $>5 \mathrm{~cm}$ \\
\hline Good response (Growth of more than $75 \%$ of terminal aesthetically acceptable hair) & $46.5 \%$ & $34 \%$ & $15 \%$ \\
\hline
\end{tabular}

Pearson measurement test analysis: $p=0.025 ; r=-0.8$.

Table 5. The hair regrowth rate according to lesion duration in the cryotherapy group.

\begin{tabular}{|c|c|c|c|}
\hline \multirow{2}{*}{ Percentage of improvement } & \multicolumn{3}{|c|}{ Lesion duration } \\
\hline & $<6$ months & 6-24 months & $>24$ months \\
\hline Good response (Growth of more than $75 \%$ of terminal aesthetic acceptable hair) & $46.5 \%$ & $37.5 \%$ & $16 \%$ \\
\hline
\end{tabular}

Pearson test analysis; $(P=0.037) ; r=-0.25$.

2. The lesions between 6 and 24 months duration: $37.5 \%$.

3. The lesions above 24 months: $16 \%$.

\section{Betamethasone group}

\section{Good response}

1. The lesions below 6 months: $48.5 \%$.

2. The lesions between 6 and 24 months duration: $32 \%$.

3. The lesions above 24 months: $18.5 \%$.

Pearson measurement test analyses showed that there was a reverse relationship between lesion duration and the rate of hair regrowth $(r=-0.25, p=0.037),(r=-0.75$, $p=0.045$ ), in the two groups sequentially. Besides that, in each group, there was a significant improvement compared to the baseline status (Tables 4 to 7 ). The response of lesions according to the diameter of patches was as follows:

\section{Group 1 (Cryotherapy)}

\section{Good response}

1. The lesions below $2.5 \mathrm{~cm}$ diameter: $45 \%$

2. The lesions between 2.5 to $5 \mathrm{~cm}$ diameter: $35.5 \%$

3. The lesions above $5 \mathrm{~cm}$ diameter: $14 \%$.

\section{Topical betamethasone group}

\section{Good response}

1. The lesions below $2.5 \mathrm{~cm}$ diameter: $46.5 \%$.

2. The lesions between 2.5 to $5 \mathrm{~cm}$ diameter: $34 \%$.

3. The lesions above $5 \mathrm{~cm}$ diameter: $15 \%$.

Pearson measurement test analyses showed that there was a reverse relationship between lesion diameter and rate of hair regrowth $(r=-0.25, \quad p=0.03), \quad(r=-0.8$, $p=0.025$ ), in the two groups sequentially (Tables 3 and 4 ). Of course, in each group there was a significant improvement compared to the baseline status.

\section{The recurrence (by definition)}

Loss of more than $50 \%$ of hairs or increase of more than $50 \%$ in the extent and size of the patches after good terminal hair regrowth (defined previously), occurred in $41 \%$ of group A and $68 \%$ of group B. On the other hand, betamethasone-treated patches were more easily prone to recurrences than cryotherapy-treated ones $\{(\mathrm{Chi}-$ Square (reliability coefficient $95 \%)(p<0.05 \%, p=0.02)\}$.

The most frequent side effect in the cryotherapy group was short term transient erythema and some mild stinging pain. There were no significant or dangerous side effects during nitrogen application or in the betamethasone treated group. Only in one patient of the 
Table 6. The improvement rate according to lesion duration in the betamethasone group.

\begin{tabular}{|c|c|c|c|}
\hline \multirow[t]{2}{*}{ Percentage of improvement } & \multicolumn{3}{|c|}{ Lesion duration } \\
\hline & $<6$ months & 6-24 months & >24 months \\
\hline Good response (Growth of more than $75 \%$ of terminal aesthetic acceptable hair) & $48.5 \%$ & $32 \%$ & $18.5 \%$ \\
\hline
\end{tabular}

Pearson test analysis: $(P=0.045) ; r=-0.75$.

Table 7. Patients demographic and lesions characteristics.

\begin{tabular}{lccc}
\hline Sex & Population (no.) & Mean age (years) & Duration (months) \\
\hline Male & 17 & 19.5 & 14 \\
Female & 22 & 24 & 9 \\
\hline
\end{tabular}

cryotherapy group was the treatment discontinued due to dissatisfaction and painful sensation. Therefore, the adverse events were mild and except for one patient; they did not warrant discontinuation of therapy. In addition, no dyspigmentation occurred in areas treated by liquid nitrogen cryotherapy at the end of this trial.

\section{DISCUSSION}

Various therapeutic agents have been described for the treatment of AA, but none are curative or preventive. Cryotherapy has also been employed to stimulate hair growth in AA (Hong et al., 2006; Hyung et al., 1994).

One study utilizing both children and adults, revealed hair regrowth in greater than $60 \%$ of affected areas in 70 of 72 patients. In that study still, after 6 months only 3 out of 66 patients had recurrences. The average size of their studied lesions was $2 \times 3 \mathrm{~cm}$ (Lei et al., 1991). In this study, $23 \%$ of lesions showed a good response (regrowth of $>75 \%$ terminal hairs) and $33.5 \%$ showed only a moderate response (that is, regrowth of 50 to $75 \%$ terminal hairs) in cryotherapy mode of treatment. The average diameters of our studied lesions were 3 to 3.5 $\mathrm{cm}$ sequentially in the two groups.

The cause of such significant difference could be racial variation of response in autoimmune diseases, criteria of inclusion for example severity of hair loss at the start of trial, or a technical difference in mode of treatment.

In another Korean study, those over 50 years of age and with more than 3 weeks of treatment interval seemed to have a relatively poor response rate. Other patient related factors such as sex and age or demographic characters were not significant. There were no significant side effects, except slight pain, swelling and erythema (Hong et al., 2006). The overall improvement rate of the Korean study was significantly higher than that of the present study.

In the opinion of Hyung et al. (1994), the overall respondents were $22(66.7 \%)$. There seemed to be good response rates of $70.0 \%$ in females and $71.4 \%$ in $\mathrm{AA}$ multiplex .These data showed a better outcome than that of the present study and the difference may be due to shorter intervals of cryo application (Hyung et al., 1994).

The best mechanisms explaining the efficacy of cryotherapy in AA are as follows:

1. Vascular changes

2. Immunomodulation

According to Hong et al. (2006), if cryotherapy is applied to diseases of the hair superficially, one can expect regrowth of the hair. The supposed mechanism is that cryotherapy dilates the vessels around hair follicles, thus improving follicular nutritional status. Their report showed that superficial cryotherapy promotes eyebrow hair growth in several patients with AA.

On the whole, nitrogen cryotherapy in patients with $A A$ can be an effective treatment. It is a simple and convenient method, and has a relatively good therapeutic response with fewer side effects. We have reported our recent experience, which shows that cryotherapy with liquid nitrogen appears to promote hair growth in patients with AA.

Cryotherapy in AA may be regarded as a safe, efficacious and easily available treatment.

\section{Conclusion}

We recommend the use of superficial cryotherapy as a helpful and practically effective treatment for patients with a mild, isolated form of $A A$, especially in children who are vulnerable to side effects of immunosuppressive drugs. Of course in future there is a plan for a longer follow up to see if there is any sustained result from cryotherapy.

\section{ACKNOWLEDGMENT}

We thank the Vice Chancellor for Research and Technology in Isfahan University of Medical Sciences for 
supporting our idea and for providing the facilities used in performing this research.

\section{REFERENCES}

Gilhar A, Ullmann Y, Berkutzki $T$ (1998). Autoimmune hair loss (Alopecia areata) transferred by $\mathrm{T}$ lymphocytes to human scalp explants on SCID mice. J. Clin. Investig. 101:62-67.

Hong SP, Jeon SY, Oh TH, Lee WS (2006). A Retrospective Study of the Effect of Superficial Cryotherapy on Alopecia areata. Korean J. Dermatol. 44(3):274-280.

Hyung OK, Seok D, Won S (1994). Effect of cryotherapy with liquid nitrogen on Alopecia areata. Korean J. Dermatol. 32(3):421-426

Lei Y, Nie Y, Zhang JM, Liao DY, Li HY, Man MQ (1991). Effect of superficial hypothermic cryosurgery with liquid nitrogen on Alopecia areata. Arch. Dermatol. 127:1851-1852.

Madani S, Shapiro J (2000). Alopecia areata update. J. Am. Acad. Dermatol. 42:549-570.
McElwee KJ (1999). Third International Research Workshop on Alopecia Areata. J. Investig. Dermatol. 112:822-824.

McElwee KJ, Spiers EM, Oliver RF (1999). Partial restoration of hair growth in the DEBR model for Alopecia areata after in vivo depletion of $\mathrm{CD}^{+} \mathrm{T}$ cells. Br. J. Dermatol. 140:432-437.

Safavi KH, Muller SA, Suman VJ (1995). Incidence of Alopecia areata in Olmstead County, Minnesota, 1975 through 1989. Mayo Clin. Proc. 70:628-633.

Schwartz RA, Janniger CK (1997). Alopecia areata. Cutis 59:238-241.

Shapiro J, Madani S (1999). Alopecia areata: Diagnosis and management. Int. J. Dermatol. 38(Suppl 1):19-24.

Sharma VK, Kumar B, Dawn G (1996). A clinical study of childhood Alopecia areata in Chandigarh, India. Ped. Dermatol. 13:372-377.

Todes-Taylor N, Turner R, Wood GS (1984). T cell subpopulations in Alopecia areata. J. Am. Acad. Dermatol. 11:216-223.

Tosti A, Morelli R, Bardazzi F, Peluso AM (1994). Prevalence of nail abnormalities in children with alopecia areata. Pediatr. Dermatol. 11: 112-115. 